\title{
ANTIDERMATOPHYTIC ACTIVITY OF STREPTOMYCES CACAOI SUBSP. CACAOI. M20
}

\author{
JANAKI T* \\ Department of Botany, K. M. Centre for P. G. Studies (Autonomous), Puducherry, India. Email: janakishaila@gmail.com
}

Received: 04 March 2017, Received and Accepted: 15 April 2017

\begin{abstract}
Objective: This study is an approach to find the alternative drug for the dermatophytosis because some of the existing antibiotics cause toxicity and also found expensive.

Methods: There were 25 actinomycetes isolated from the soil sample of the mangrove, Avicennia marina from Ariyankuppam backwater area, Puducherry. Among the 25 isolates, the most active isolate, M20 was selected initially based on its antifungal activity, and it was checked for antidermatophytic activity against three dermatophytes, Microsporum gypseum, Trichophyton mentagrophytes, and Epidermophyton floccosum by agar plug method, well diffusion method followed by food poisoning technique. Release of volatile compounds from the isolate M20 has also been tested. Ultraviolet (UV)-vis spectral analysis of partially purified yellow compound fraction was done.

Results: The isolate M20 actively controlled the growth of dermatophytes by agar plug method in the primary screening, well diffusion method in the secondary screening. The isolate was identified as Streptomyces cacaoi sub sp. cacaoi by 16 sRNA sequencing analysis. The $10 \%$ (10 ml) culture filtrate of the isolate M20 was found to control the radial growth of these three dermatophytic fungi by food poisoning technique. Volatile compounds of the isolate M20 affected the mycelial growth of M. gypseum tremendously. Complete arrest of growth of M. gypseum was noticed with the combination of both volatile and non-volatile antibiotic compound of the isolate M20. The partially purified compound fraction (yellow) ( $0.5 \mathrm{mg} / 6 \mathrm{~mm}$ disc) inhibited the growth of M. gypseum (12 mm), and it was compared with the standard antibiotic clotrimazole ( $0.5 \mathrm{mg} / 6 \mathrm{~mm}$ disc), whose inhibition on
\end{abstract} M. gypseum was only $6 \mathrm{~mm}$. UV-vis spectral analysis revealed the compound belonged to nucleoside antibiotics.

Conclusion: It is evident that the mangrove actinomycetes are potential for preparing biomedicines for human welfare.

Keywords: Mangrove actinomycetes, Dermatophytosis, Streptomyces cacaoi sub sp. cacaoi.

(C) 2017 The Authors. Published by Innovare Academic Sciences Pvt Ltd. This is an open access article under the CC BY license (http://creativecommons. org/licenses/by/4. 0/) DOI: http://dx.doi.org/10.22159/ajpcr.2017.v10i7.18270

\section{INTRODUCTION}

In recent years, $20-30 \%$ of people are affected by dermatophytosis caused by three fungal genera, i.e., Microsporum, Trichophyton, and Epidermophyton. These fungi feed on keratin of hair, skin, and nail. Serious consequences of dermatophytosis are uncommon and infections can be self-limiting. The illness may be disfiguring and uncomfortable, especially when the lesions are widespread. There are many antifungal antibiotics obtained from fungi and bacteria, and these are more common, antidermatophytic compounds from actinomycetes especially Streptomyces spp. [1-5], Streptosporangium spp. [6] have been intensively studied. Recently, the extraction of antibiotics from mangrove actinomycetes has been catalyzed in finding better antibiotics for dermatophytosis that are less expensive and less or no side effects. Studies related to antifungal, antibacterial, and anticandida activity have been regularly reported from mangrove actinomycetes [7-11]. Hence, definitely, the mangrove actinomycetes can be a source for extracting novel antidermatophytic compounds for dermatophytes. This is an approach to find the antidermatophytic activity and taken an initial step for better drug development for dermatophytosis from actinomycetes from mangroves of Ariyankuppam backwater area in Puducherry.

\section{METHODS}

Collection of soil sample and soil analysis

The dominant mangrove plant in Ariyankuppam backwater area of Puducherry (Lat $11^{\circ} 4603^{\prime \prime}$ to $11^{\circ} 53^{\prime} 40^{\prime \prime}$ North and Longi $79^{\circ} 49^{\prime} 45^{\prime \prime}$ to $79^{\circ} 48^{\prime} 00^{\prime \prime}$ East) is Avicennia marina (Forsk). Vierh - (Avicenniaceae). Soil sample was collected near the root region of the mangrove plant, and it was air dried for 1 week at $40^{\circ} \mathrm{C}$. Physio-chemical nature of soil sample was analyzed in Soil Testing Laboratory, Department of Agriculture, Puducherry, India.
Isolation of mangrove actinomycetes

The soil sample was subjected to dry heat $\left(70^{\circ} \mathrm{C}\right.$ for 15 minutes $)[12,13]$ pretreatment. $0.1 \mathrm{ml}$ of last two serial dilutions $\left(10^{-5}\right.$ and $\left.10^{-6}\right)$ was inoculated by pour plate method [14] using starch casein agar supplemented with fluconazole $80 \mu \mathrm{g} / \mathrm{ml}$ and nalidixic acid $75 \mu \mathrm{g} / \mathrm{ml}$. Plates were incubated at $30 \pm^{\circ} \mathrm{C}$ for up to 30 days. The selected colonies were maintained in yeast malt extract agar slants.

\section{Test organisms used in this study and their preparation}

Microsporum gypseum (microbial type culture collection [MTCC]-4494), Trichophyton mentagrophytes (MTCC-8476), and Epidermophyton floccosum (MTCC-7880) are procured from MTCC-Chandigarh. Test pathogens were maintained in potato dextrose agar slants and plates, $\mathrm{pH}-6.5$. These were stored in a refrigerator at $4^{\circ} \mathrm{C}$ for future use. 5-7 days old cultures were used for antidermatophytic study.

In vitro screening for antidermatophytic activity

Initially, the antidermatophytic activity was tested with 25 isolates of actinomycetes by agar plug method $[15,16]$. Among the 25 isolates, the isolate M20 found to be better in controlling all the three dermatophytes tested. Hence, the isolate M20 was selected for secondary screening by agar well diffusion method [17]. Among the three dermatophytes tested against mangrove actinomycetes for antidermatophytic activity, the dermatophyte $M$. gypseum found grow better in potato dextrose agar, nutrient agar than compared to T. mentagrophytes and E. floccosum.

\section{Biochemical and molecular characterization of isolate M20}

Production of extracellular enzyme chitinase [18] was tested, to find chitin degradation in the cell wall of fungal pathogens. For the 16sRNA sequencing analysis of isolate M20, the purified PCR products of approximately $1,400 \mathrm{bp}$ were sequenced using two universal primers: 
518F 5'CCAGCAGCCGCGGTAATACG 3', 800R 5' TACCAGGGTATCTAATCC 3 '. The isolate M20 was identified.

\section{Effect of culture filtrate of isolate M20 on radial growth of} M. gypseum

Food poisoning technique [19] was followed to study the effect of culture filtrate of M20 on radial growth of M. gypseum, $10 \mathrm{ml}$ culture filtrate was added to $90 \mathrm{ml}$ of molten potato dextrose agar $(\mathrm{pH} \mathrm{6}$ ) to get a final concentration of $10 \%$. Now the $100 \mathrm{ml}$ potato dextrose agar medium was ready to serve five sterilized Petri plates with dia $90 \mathrm{~mm}$. PDA Petri plates without $10 \%$ culture filtrate were kept as a control for the test fungal pathogen-M. gypseum. All the plates were then inoculated in the center with $8 \mathrm{~mm}$ dia. Mycelial plug cut from actively growing plate culture of $M$. gypseum. All the plates were incubated under room temperature $28 \pm 2^{\circ} \mathrm{C}$ for $4-5$ days. Growth of the fungal pathogen in treated plates was measured when the growth of the organism in control plate was full. The percentage inhibition in mycelial growth was calculated using the standard equation given below.

Inhibition of mycelia growth in $\%=\frac{\text { Control }- \text { treatment }(10 \%)}{\text { Control }} \times 100$

\section{Production of volatile compounds}

Production of volatile compounds by the isolate of the M20 was assessed by inverted plate technique [20]. Six modified nutrient glucose agar plates ( $\mathrm{pH} 7.5$ ) were inoculated with the spore suspension of isolate M20 and incubated for 10 days. After 10 days, another set of six PDA Petri plates was inoculated center with mycelial disc of the test pathogen M. gypseum. Then, the base of the plates containing the test fungi was placed upside down on the basal plates containing the growth of isolate M20. Plates were sealed with adhesive tape to prevent the escape of volatile factors. The sealed plates were incubated at room temperature along with a suitable control for 5 days. The growth measurements were made after 5 days. In another set of Petri plates, PDA amended with culture filtrate $10 \%$ of isolate M20 was used to test the combined effect of volatile as well as non-volatile antibiotics on the mycelial growth of the pathogen.

Partial purification of methanolic crude extract by column chromatography

Using chloroform: Methanol: Acetic acid (8.5:1.5: $0.2 \mathrm{ml})$ as the mobile phase two color spots were resolved in both in ascending paper chromatography and TLC. Since methanol extraction produced the highest activity, the culture filtrate (1 l) was extracted in methanol, after drying, produced six gram of dark brown oily residue. The residue was mixed with silica gel and applied to the silica gel column (230-400 mesh) and eluted with chloroform: Methanol: Acetic acid (85:15:2 ml) to give two active fractions. The yellow compound fraction was used for further investigation.

Antidermatophytic activity of the partially purified compound fraction against $M$. gypseum with reference standard antimicrobial agent clotrimazole

The isolate M20 inhibited the growth of dermatophytes better by agar plug, well diffusion method and food poisoning method. The efficiency of inhibition of dermatophytes by partially purified compound fraction was tested for antidermatophytic activity against M. gypseum.

Antibiotic activity of the partially purified methanol compound fraction was compared with the standard antibiotic clotrimazole discs. Sterile $6 \mathrm{~mm}$ Whatman No.1 paper disc with $0.5 \mathrm{mg}$ of clotrimazole was prepared. Sterile $6 \mathrm{~mm}$ Whatman No. 1 paper discs loaded with $0.5 \mathrm{mg}$ of partially purified compound fraction in methanol and control discs loaded $25 \mu \mathrm{l}$ of methanol was used for bioassay against M. gypseum. The plates were incubated under the room temperature for 24-72 hrs. The development of inhibition zone around the discs was measured and recorded after the $24 \mathrm{hrs}$ of incubation.
Minimum inhibitory concentration (MIC)

MIC of the partially purified compound fraction was determined using the test fungi by tube dilution method [21].

$1 \mathrm{ml}$ portions of potato dextrose broth were amended with the sample to get $10 \mu \mathrm{g}, 20 \mu \mathrm{g}, 30 \mu \mathrm{g}, 40 \mu \mathrm{g}, 50 \mu \mathrm{g}, 75 \mu \mathrm{g}$, and $100 \mu \mathrm{g} / \mathrm{ml}$. One set of potato dextrose broth tubes without any addition was maintained as control and another set amended with $100 \mu \mathrm{g} / \mathrm{ml}$ of clotrimazole served as positive control. All the tubes were inoculated with a loop full of spores of M. gypseum, mixed well and incubated at $30^{\circ} \mathrm{C}$ for $24 \mathrm{hrs}$. After $24 \mathrm{hrs}$, the growth level in each tube was noted; cultures from all tubes were aseptically streaked onto potato dextrose agar plate using sterile cotton swabs and incubated at $30^{\circ} \mathrm{C}$ for $72 \mathrm{hrs}$. The growth inhibition of M. gypseum was recorded after $72 \mathrm{hrs}$.

Ultraviolet (UV)-visible spectral analyses of methanolic crude extract of isolate M20

UV-visible spectral analysis of methanol crude extract of isolate M20 was performed using Hitachi U-2010 spectrophotometer, wavelength range: $200-800 \mathrm{~nm}$ to know the group of the compounds responsible for antidermatophytic activity.

\section{RESULTS AND DISCUSSION}

\section{Collection and analysis of soil sample}

The mangrove plant A. marina in Ariyankuppam backwater area in Puducherry, showed high tolerance with ethnobotanical medicinal importance to survive in the saline environment. The diversity of microbes in root region of plant highly depends on the bioactive compounds that are secreted and released out by the plant for its survival. The interaction of rhizosphere actinomycetes with plant is coordinated by the root exudates. The wet $\mathrm{pH}$ of mangrove sediment sample collected from A. marina was 7.7. The physicochemical analysis of soil sample showed that there were very low available Nitrogen, $\mathrm{P}_{2} \mathrm{O}_{5}$, and $\mathrm{Cu}$. $\mathrm{K}_{2} \mathrm{O}$ was medium. Micronutrients such as $\mathrm{Zn}$ and Fe were high in their available form, Mn was medium.

Isolation and in vitro screening of mangrove actinomycetes for antidermatophytic activity

The air-dried soil sample had reduced the unwanted bacteria and fungi initially. The soil sample pretreated with dry heat $\left(70^{\circ} \mathrm{C}\right.$ for 15 minutes) [12,13] pretreatment had eliminated unwanted bacteria and fungi further, and also it enhanced the chances of isolating rare and novel actinomycetes [22]. Yeast malt extract agar was found better in the maintenance of actinomycetes that produced spores.

When the 25 actinomycetes were subjected for primary screening against the three dermatophytes, namely, M. gypseum, T. mentagrophytes, and E. floccosum, the isolate M20 was found to better in controlling all the three dermatophytes (Plate 1). Isolate M20 not only controlled these three dermatophytes but also broad spectrum antifungal activity was observed and noted against 12 fungi tested in the earlier study [10]. A polyene belonging to the pentaene group which has a broad spectrum antifungal activity in vitro against many fungal strains including dermatophytes was extracted from Streptomyces griseus strain [1]. Novel antibiotics UK-2A, B, C, and D produced by Streptomyces sp. 517-02 exhibit strong antifungal

Table 1: Effect of culture filtrate of M20 of radial growth of dermatophytes

\begin{tabular}{lll}
\hline \multirow{2}{*}{ Fungi tested } & \multicolumn{1}{l}{$\mathbf{1 0 \%}$ culture filtrate } \\
\cline { 2 - 3 } & Growth in $\mathbf{~ m m}$ & Inhibition in $\mathbf{~ m m}$ \\
\hline Microsporum gypseum & $4.4 \pm 0.2$ & $85.6 \pm 0.2$ \\
Trichophyton mentagrophytes & $4.4 \pm 0.2$ & $85.6 \pm 0.2$ \\
Epidermophyton floccosum & $8.9 \pm 0.3$ & $81.1 \pm 0.3$ \\
\hline
\end{tabular}

The data are presented as mean \pm value standard deviation of three replicates $(n=3)$ 
activity [23]. Streptomyces halstedii K122 found to produce antifungal compounds on solid substrates that inhibited the radial growth of dermatophytic fungi [24].

Biochemical and molecular characterization of isolate M20 The broad spectrum antifungal activity not only depends on the compounds in the form of antibiotics but also mainly depends on the extracellular enzyme production by isolates. When the isolate M20 was tested for chitinase production, it was found better in the production of chitinase enzyme that may also responsible for cell wall degeneration in fungi. The study about chitinase production by Streptomyces sp got its support from the studies $[25,26]$. Molecular characterization of isolate M20 was done by $16 \mathrm{~S}$ rRNA sequencing analysis. The sequence of isolate M20 was submitted to GeneBank with the accession No. KP872910. Phylogenetic analysis of 16S rRNA gene (1427 bp) of M20, species of Streptomyces was carried out with 18 different reference species of Streptomyces available in the GeneBank database. The isolate M20 branched along with Streptomyces cacaoi subsp cacaoi (NRBC 12748(T)-AB184115 in the analysis.

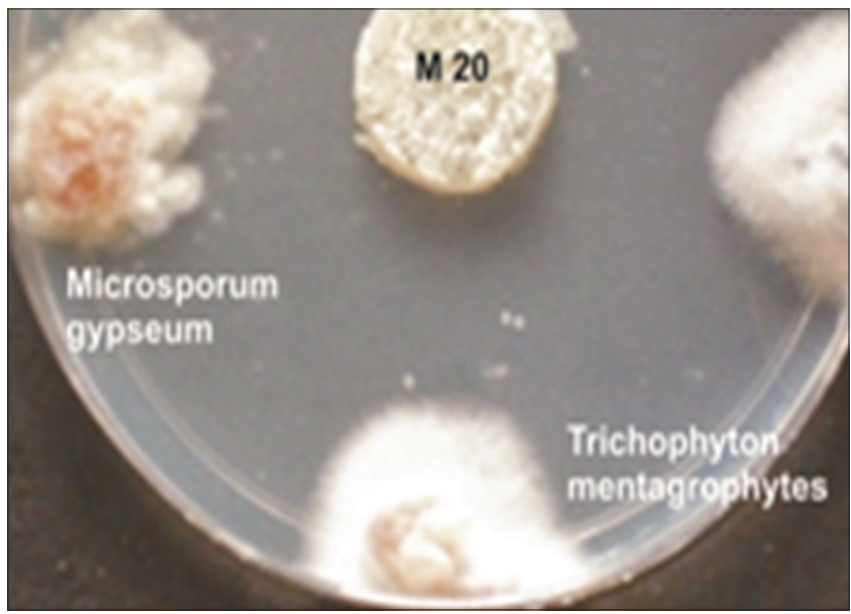

Plate 1: Antidermatophytic activity of isolate M20 by agar plug method
Antidermatophytic activity - Effect of culture filtrate of M20 on radial growth of dermatophytes

Control of three dermatophytes was initially tested under in vitro condition using agar plug method, agar well diffusion method. $10 \%$ culture filtrate of isolate M20.

Inhibition in radial growth of test fungi was observed and noted as M. gypseum 95.6\%, T. mentagrophytes $95.6 \%$, and E. floccosum $91.1 \%$ (Plate 2).

It was evidenced that the $10 \%$ culture filtrate had effectively controlled the dermatophytes (Table 1) and also noted that the inhibition in radial growth of fungi tested for the activity was stable up to 10-15 days.

\section{Production of volatile compounds of the M20}

The volatile compounds produced by the isolate M20 was tested with M. gypseum. It was noticed that the presence of volatile compounds adversely affected the growth and mycelial mass of fungi as $76.2 \%$. The combination of volatile toxicity of $\mathrm{M} 20+10 \%$ culture filtrate enhanced the inhibition of as $99.8 \%$ (Plate 3). The effectiveness of volatile compounds with non-volatile antibiotic compounds on the mycelial growth of test pathogen proved the suppressing capacity of isolate M20. From these, it was clear that the volatile and non-volatile compounds secreted by the isolate M20 actively controlled the growth of dermatophyte - M. gypseum.

Antidermatophytic activity of the partially purified compound fraction against $M$. gypseum with reference standard antibiotic clotrimazole

Antidermatophytic activity was initially tested with isolate M20 against three dermatophytes M. gypseum, T. Mentagrophytes, and E. floccosum by agar plug and agar well diffusion method. The activity was found to better in both the methods. Since the culture filtrate of isolate M20 was found to be better, the activity was tested with the partially purified compound fraction that was extracted from the culture filtrate. Antidermatophytic activity of the partially purified compound fraction $(0.5 \mathrm{mg} / \mathrm{disc})$ with reference standard antimicrobial agent clotrimazole $(0.5 \mathrm{mg} / \mathrm{disc})$ was tested against M. gypseum. The inhibition zone in antidermatophytic activity of compound fraction was better $(12 \mathrm{~mm})$ than the clotrimazole $(6 \mathrm{~mm})$ tested (Plate 4$)$.
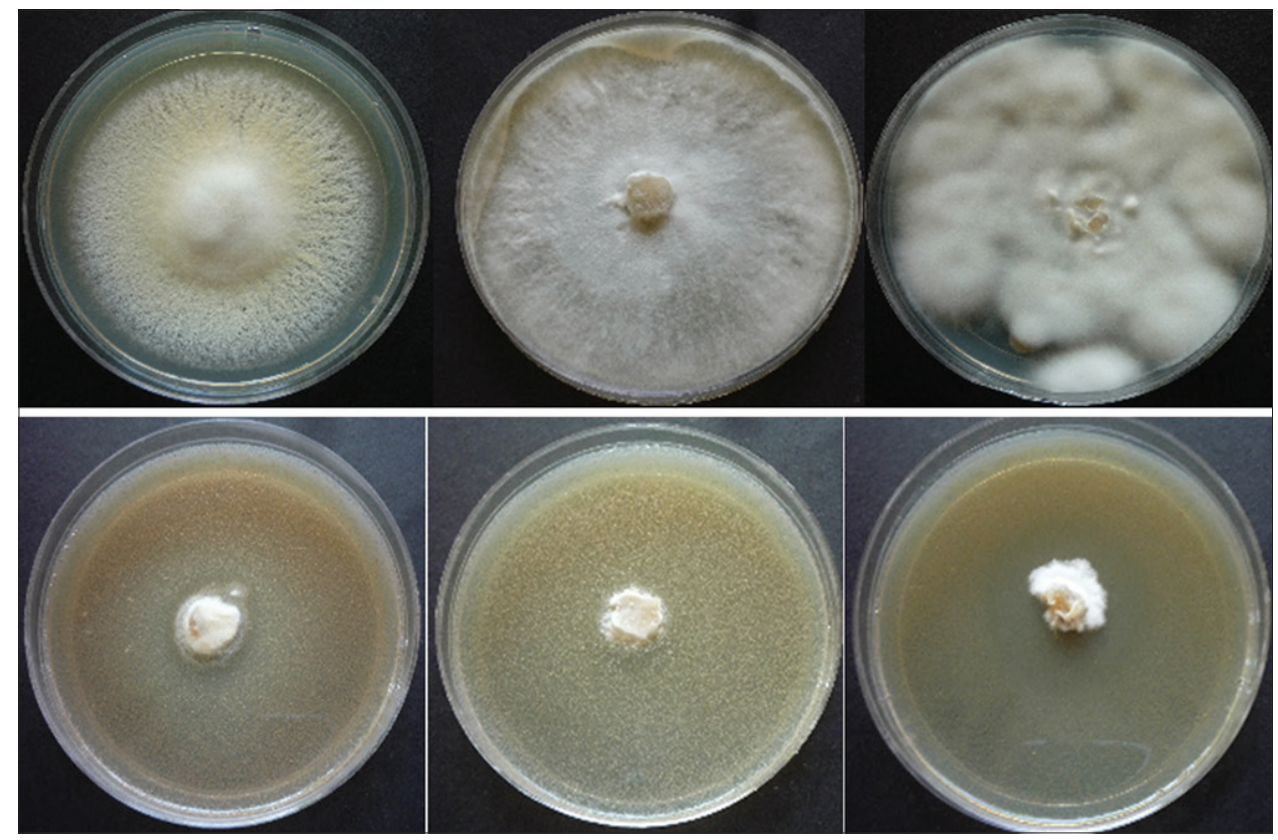

Plate 2: Effect on 10\% culture filtrate of isolate M20 on radial growth of dermatophytes control Microsporum gypseum Trichophyton mentagrophytes Epidermophyton floccosum treated with $10 \%$ culture filtrate of isolate M20 M. gypseum T. mentagrophytes, E. floccosum 


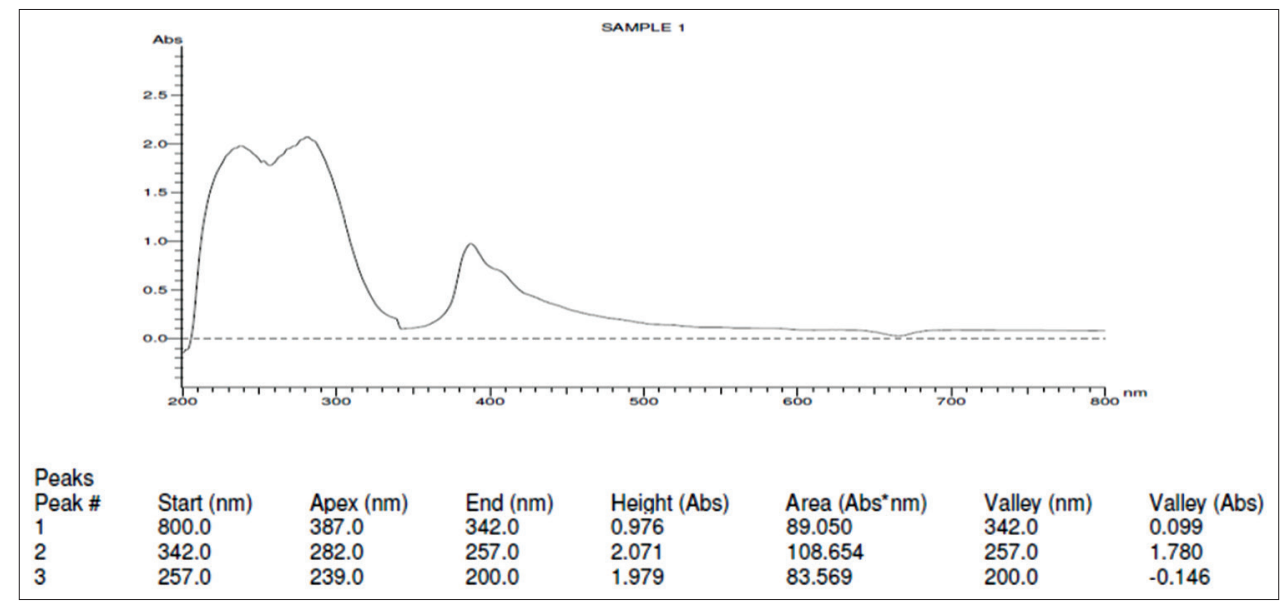

Fig. 1: Analysis of Ultraviolet- visible spectrum of partially purified yellow compound fraction
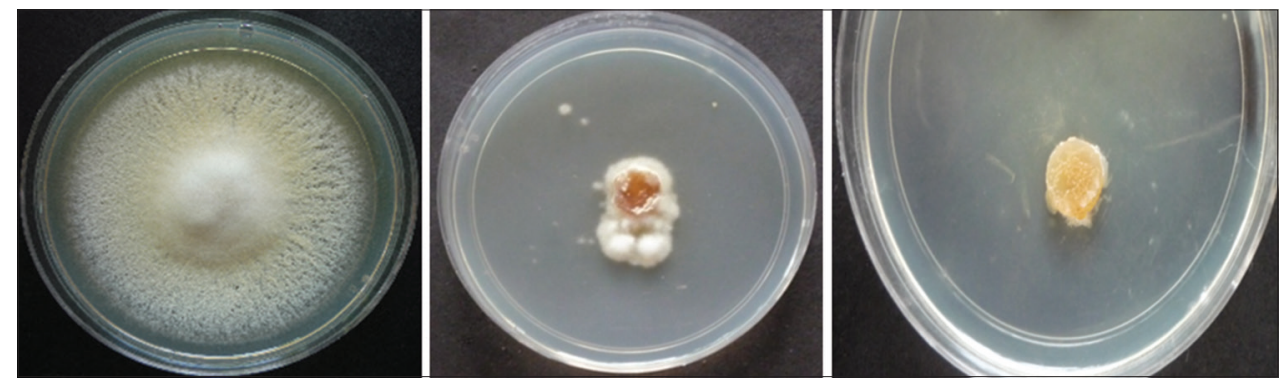

Plate 3: Effect of volatile compounds of the isolate M20 on Microsporum gypseum control volatile toxicity of M20 volatile toxicity of M20+10\% C.F on M. gypseum on M. gypseum antidermatophytic activity of the partially purified compound fraction against $M$. gypseum with reference standard antibiotic clotrimazole

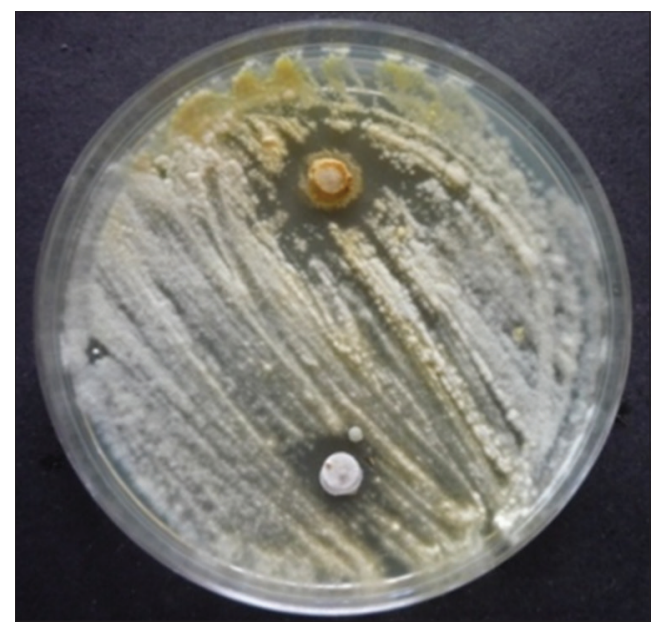

Plate 4: Antidermatophytic activity of the partially purified compound fraction with clotrimazole against Microsporum gypseum

Minimum inhibitory concentration (MIC)

MIC of partially purified compound fraction of isolate M20 for antidermatophytic activity against M. gypseum was $75 \mu \mathrm{g} / \mathrm{ml}$. The complete antidermatophytic activity was observed with the concentration of $100 \mu \mathrm{g} / \mathrm{ml}$ (Plate 5).

UV-visible spectrum of partially purified yellow compound fraction The presence of neutral and acidic polyoxins (270-290 nm) and heptaene antifungal antibiotics (385-417 nm) are confirmed from the UV-vis spectral analysis with the help of absorption spectra of reference antibiotics [27]. The nucleoside antibiotics are very effective in broad spectrum antifungal, antibacterial, anticandida activity

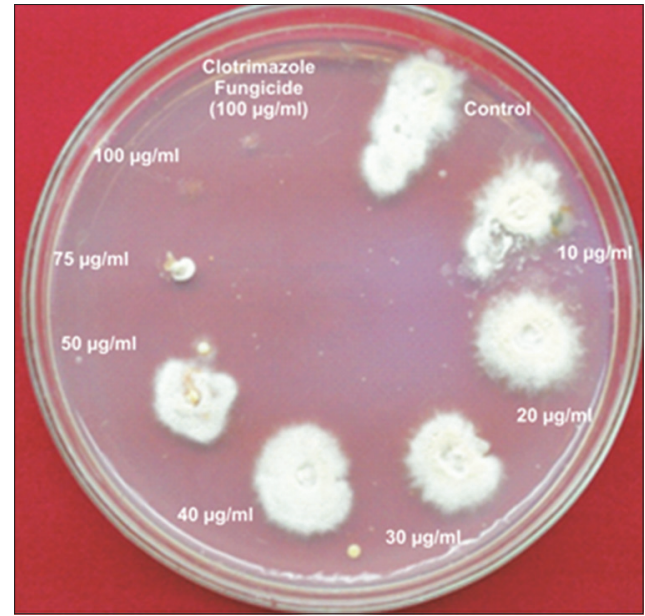

Plate 5: Detection of minimum inhibitory concentration for Microsporum gypseum from broth dilution technique

and also they have insecticidal, nematocidal, and larvicidal antiviral properties. Hence, this group of antibiotics used for curing and controlling multiple diseases in humans and animals. Since they have strong antifungal activity including antidermatophytic activity, they can be used to prepare biomedicines for human and animal welfare (Fig. 1).

\section{CONCLUSIONS}

Every day human pathogens are getting resistance to the drugs to tackle the problem of getting rid of them. Hence, they enhance their resistive virulence with the help of suitable environmental conditions, food habit of humans and over usage of antibiotics. This leads to search of new and newer antibiotics to protect ourselves from the human pathogens. 
The dermatophytes do not cause any serious problems in human beings, and other animals but disfiguring and uncomfortable are common. This can be controlled by the antifungal antibiotics that are already available in the market but some antibiotics are expensive and most cause severe side effects. This experimental research work is an approach to find the efficiency of antidermatophytic activity of culture filtrate, partially purified compound fraction of isolate M20 to proceed further toward the alternative drug from mangrove actinomycetes for dermatophytosis. It is a preliminary work, needs further research work for better drug delivery for dermatophytosis.

\section{REFERENCES}

1 Staib F, Mishra SK, Formtling RA, Blisse A, Kleinkauf FH, Mahmutoglu I, et al. A new polyene with antimycotic activity produced by Streptomycetes from the rhizosphere of an indoor plant. Arzneimette Lforschung 1983;33(1):84-7.

2 Kotake C, Yamasaki T, Moriyama T, Shinoda M, Komiyama N, Furumai $\mathrm{T}$, et al. Butyrolactols $\mathrm{A}$ and $\mathrm{B}$, new antifungal antibiotics. Taxonomy, isolation, physico-chemical properties, structure and biological activity. J Antibiot (Tokyo) 1992;45(9):1442-50.

3 Chattopadhyay D, Sen SK. Optimisation of cultural conditions for antifungal antibiotic accumulation by Streptomyces rochei G164. Hindustan Antibiot Bull 1997;39(1-4):64-71.

4 Raytapadar S, Paul AK. Production of an antifungal antibiotic by Streptomyces aburaviensis 1DA-28. Microbiol Res 2001;155(4):315-23.

5 Yassin MH, Ghaly MF, Shash SM, El-Doraidy EH. Potentiality of actinomycete isolates against dermatophytic fungi, 1-Biological, taxonomical studies and environmental factors affecting the antifungal production of Streptomyces kanamyceticus EHE-68. N Egypt J Microbiol 2005;2:45-62.

6 Hacène $\mathrm{H}$, Boudjellal $\mathrm{F}$, Lefebvre $\mathrm{G}$. AH7, a non-polyenic antifungal antibiotic produced by a new strain of Streptosporangium roseum. Microbios 1998;96(384):103-9.

7 Bhattacharya S, Nag C, Das A. Evaluation of antagonistic activities of microbes from Vallapattanam and Pappinishery mangrove ecosystems of Kannur district in Kerala, India. Int J Pharm Life Sci 2012;3(5):650-1659.

8 Das A, Bhattacharya S, Yegoup A, Mohammed H, Rajan SS. In vitro antimicrobial activity and characterization of mangrove isolates of Streptomycetes effective against bacteria and fungi of nosocomial origin. Braz Arch Biol Technol 2014;57(3):349-56.

9 Janaki T, Nayak BK, Ganesan T. Antibacterial activity of soil actinomycetes from the mangrove Avicennia marina. J Pharmacogn Phytochem 2016a;5(1):267-71.

10 Janaki T, Nayak BK, Ganesan T. Antifungal activity of soil actinomycetes from the mangrove Avicennia marina. J Med Plants Stud 2016b;4(2):5-8.

11 Janaki T, Nayak BK, Ganesan T. Screening mangrove actinomycetes for anticandida activity. Pharm Innov J 2016g;5(7):29-35.

12 Hayakawa M, Sadaka T, Kayiura T, Nonomura H. New methods for the highly selective isolation Micromonospora and Microbispora. J Ferment Bioeng 1991;72:320-6.

13 Janaki T, Nayak BK, Ganesan T. Different pre-treatment methods in selective isolation of actinomycetes from mangrove sediments of Ariyankuppam, back water estuary, Puducherry. Int J Adv Res Biol Sci 2014b;1(6):154-63.

14 Zheng Z, Zeng W, Huang Y, Yang Z, Li J, Cai H, et al. Detection of antitumor and antimicrobial activities in marine organism associated actinomycetes isolated from the Taiwan Strait, China. FEMS Microbiol Lett 2000;188(1):87-91

15 Mohanraj D, Bharathi S, Radhakrishnan M, Balagurunathan R. Bioprospecting of actinobacteria from Yelagiri hills with special reference to antibacterial activity. J Chem Pharm Res 2011;3(3):439-46.

16 Janaki T, Nayak BK, Ganesan T. Antibacterial activity of soil actinomycetes from the mangrove Avicennia marina. J Pharmacogn Phytochem 2016c;5(1):267-71

17 Murray PR, Baron EJ, Pfaller MA, Tenover FC, Yolken HR. Manual of Clinical Microbiology. $6^{\text {th }}$ ed. Washington, DC: ASM Press; 1995. p. 15-8.

18 Hsu SC, Lockwood JL. Powdered chitin agar as a selective medium for enumeration of actinomycetes in water and soil. Appl Microbiol $1975 ; 29(3): 422-6$

19 Grover RK, Moore JD. Toximetric studies of fungicides against brown rot organism Scelerotina fruitycola. Phytopathology 1962;52:876-80.

20 Bruce A, Austin WJ, King B. Control of growth of Lentinus lepideus by volatiles from Trichoderma. Br Mycol Soc 1984;88(3):503-13.

21 David C, Copper EL. Mode of the action of quinolones. Rev Infect Dis 1988;10:14-9.

22 Janaki T, Nayak BK, Ganesan T. Antibacterial activity of mangrove actinomycetes isolated by eight different pre-treatment methods from backwater estuary, Ariyankuppam, Puducherry. Int J Pharm Res Bio 2014c;3(6):132-49.

23 Hanafi M, Shibata K, Ueki M, Takniguchi M. Novel antifungal antibiotics UK-2A, B, C and D from Sterptomyces sp. 5217-5302. J Antibiot (Tokyo) 1996;49(12):1226-31.

24 Frändberg E, Petersson C, Lundgren LN, Schnürer J. Streptomyces halstedii $\mathrm{K} 122$ produces the antifungal compounds bafilomycin $\mathrm{B} 1$ and C1. Can J Microbiol 2000;46:753-8.

25 Thirumurugan D, Sankari D, Vijayakumar R. Screening of chitinase production and antifungal activity of Streptomyces Sp. Act7 from east coast region, South India. Int J Pharm Pharm Sci 2015;7(5):38-41.

26 Janaki T. Biocontrol of Fusarium oxysporum in unsterilized soil by novel Streptomyces cacaoi subsp cacaoi [M20]. Int J Pharm Pharm Sci 2017;9(3):78-83

27 Khan M, Solanki R, Lal R. Selective isolation of rare actinomycetes producing novel antimicrobial compound. Int $\mathrm{J}$ Adv Biotech Res 2011;2(3):357-75. 